\title{
ON COMPLETELY MONOTONE FUNCTIONS
}

\section{$\mathrm{ON} C_{+}(X)$}

\section{J. HOFFMANN-JØRGENSEN and P. RESSEL}

\section{Introduction.}

Let $X$ be a completely regular Hausdorff space, let $C(X)$ denote the vector space of all bounded realvalued continuous functions on $X$ and $M(X)$ the vector space of all real Radon measures on $X$. The positive cones in $C(X)$ and $M(X)$ are denoted by $C_{+}(X)$ and $M_{+}(X)$.

Under pointwise addition the cone $C_{+}(X)$ becomes a 2-divisible abelian semigroup in the sense of [1]. As in [1] we define the character semigroup $\hat{S}$ of $S:=C_{+}(X)$ by $\varrho \in \hat{S}$ if and only if $\varrho: S \rightarrow[0,1]$ and

$$
\begin{gathered}
\varrho(0)=1 \\
\varrho(f+g)=\varrho(f) \varrho(g) \quad \forall f, g \in S .
\end{gathered}
$$

In the topology of pointwise convergence and with pointwise multiplication $\hat{S}$ becomes a compact topological abelian semigroup.

Let $L$ denote all functionals $\lambda: C_{+}(X) \rightarrow[0, \infty]$ satisfying

$$
\lambda(0)=0
$$

and

$$
\lambda(f+g)=\lambda(f)+\lambda(g) \quad \forall f, g \in C_{+}(X) .
$$

Each $\lambda$ satisfying (1.3) and (1.4) is increasing and hence positive homogeneous, i.e.

$$
\lambda(a f)=a \lambda(f) \quad \forall a \geqq 0, \forall f \in C_{+}(X),
$$

with the usual conventions $0 \cdot \infty=0$ and $a \cdot \infty=\infty, \forall a>0$. Equipped with the topology of pointwise convergence $L$ becomes compact and

$$
\lambda(\cdot) \mapsto e^{-\lambda(\cdot)}
$$

is a homeomorphism of $L$ onto $\hat{S}$.

Received June 4, 1976. 
We shall consider the following subsets of $L$ :

$$
L_{0}:=\left\{\lambda \in L \mid \lambda(f)<\infty, \forall f \in C_{+}(X)\right\}
$$

and, if $Y \subseteq X$

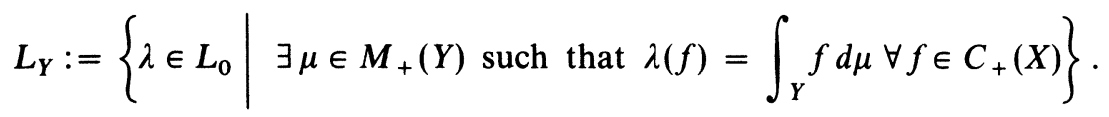

Let $w^{*}$ be the weak topology on $M(X)$, that is, $w^{*}=\sigma(M(X), C(X))$, then the map

$$
\mu \mapsto \int_{X} \cdot d \mu
$$

is a homeomorphism of $M_{+}(X)$ onto $L_{X}$.

Let $X$ denote the Stone-Cech compactification of $X$ and let $\widetilde{f}$ denote the unique continuous extension of $f$ to $\beta X$, for all $f \in C(X)$. Then the map

$$
\tilde{\mu} \mapsto \int_{\beta X} \tilde{\sim} d \tilde{\mu}
$$

is a homeomorphism of $\left(M_{+}(\beta X), w^{*}\right)$ onto $L_{0}$.

A function $\varphi: C_{+}(X) \rightarrow R$ is completely monotone if and only if $\varphi$ is bounded and

$$
\sum_{i=j}^{n} \sum_{j=1}^{n} c_{i} c_{j} \varphi\left(f_{i}+f_{j}\right) \geqq 0
$$

for all $n \in \mathrm{N}, c_{1}, \ldots, c_{n} \in \mathrm{R}$ and $f_{1}, \ldots, f_{n} \in C_{+}(X)$, (cf. [2] and Theorem 4.2 in [1]). From [2] we know that every completely monotone function $\varphi$ : $C_{+}(X) \rightarrow R$ has a unique representing measure $\xi \in M_{+}(L)$ in the sense that

$$
\varphi(f)=\int_{L} e^{-\lambda(f)} d \xi(\lambda), \forall f \in C_{+}(X) .
$$

Our aim in the following will be to establish a connection between continuity properties of $\varphi$ and the concentration of the measure $\xi$ to some "nice" subsets of $L$. A very special result of this type has already been proved in Theorem 6.1 of [1]. There $X$ is the finite set $\{1, \ldots, p\}$ with discrete topology, $C_{+}(X)$ can be identified with $R_{+}^{p}$ and $L$ with $[0, \infty]^{p}$ and it is shown that a completely monotone functions on $R_{+}^{p}$ is continuous if and only if the representing measure is concentrated on $R_{+}^{p}$.

If we consider the dual pair $(C(X), M(X))$, two natural topologies on $C(X)$ arise, the weak topology, denoted by $w$, and the Mackey topology, which we 
shall denote by $m$. We shall need two further topologies. First we define the $L_{1}$ topology $\tau$ on $C(X)$ by the family of seminorms

$$
r_{K, \sigma}(f):=\int_{K}\left|\int_{X} f d \mu\right| d \sigma(\mu)
$$

where $K$ runs through all $w^{*}$-compact, uniformly tight subsets of $M(X)$ and $\sigma$ runs through $M_{+}\left(M(X), w^{*}\right)$. There is a simpler description of this topology, but first we need a lemma:

Lemma 1.1. Lèt $\sigma \in M_{+}\left(M(X), w^{*}\right)$ and suppose that the function $\mu \mapsto|\mu|(X)$ is $\sigma$-integrable, then

$$
\lambda(A):=\int_{M(X)}|\mu|(A) d \sigma(\mu), \quad A \in \mathscr{B}(X)
$$

is a positive finite $\tau$-smooth measure on $(X, \mathscr{B}(X))$, and for every bounded Borel functions $\mathrm{g}$ on $\mathrm{X}$ we have

$$
\int_{X} g d \lambda=\int_{M(X)}\left(\int_{X} g d|\mu|\right) d \sigma(\mu) .
$$

If $\sigma$ satisfies

$$
\sigma(M(X))=\sup \{\sigma(K) \mid K \text { uniformly tight and closed }\}
$$

then $\lambda$ is a Radon measure on $X$.

REMARK. $\mathscr{B}(X)$ of course denotes the Borel $\sigma$-algebra of $X$. The notion of $\tau$ smoothness may be found in [7, p. XII], and from P 16 p. XIII in [7] it follows that if $X$ can be homeomorphically embedded as a universally measurable subset of a compact space $Y$, then every $\tau$-smooth finite measure on $X$ is a Radon measure (e.g. if $X$ is analytic, or if $X$ is $\sigma$-compact, or if $X$ is locally compact or if $X$ is complete in the sense of Čech). From Proposition 1 in [5] we know that the function $\mu \mapsto|\mu|(A)$ is Borel on $M(X)$ for every Borel set $A \subseteq X$, it is lower semicontinuous if $A$ is open.

Proof of Lemma 1.1. In the first part we only need to show $\tau$-smoothness of $\lambda$. Let a collection of open sets $G_{\alpha} \subseteq X$ filter up to $G$. Then the lower semicontinuous functions $\mu \mapsto|\mu|\left(G_{\alpha}\right)$ filter up to $\mu \mapsto|\mu|(G)$ and applying P 15 of [7] we get $\lambda(G)=\sup \lambda\left(G_{a}\right)$.

The second part is proved in a straightforward manner, taking into account that $\lambda$ is inner regular w.r.t. the closed subsets of $X$, cf. P 16 in [7]. 
Corollary 1.2. The $L_{1}$-topology $\tau$ on $C(X)$ is generated by the seminorms

$$
q_{\mu}(f):=\int_{X}|f| d \mu
$$

where $\mu$ runs through $M_{+}(X)$.

Proof. Let $K$ be a $w^{*}$-compact and uniformly tight subset of $M(X)$ and $\sigma$ a positive finite Radon measure on $M(X)$, then

$$
\mu(A):=\int_{K}|v|(A) d \sigma(v) \quad A \in \mathscr{B}(X)
$$

is a finite positive Radon measure on $X$ by Lemma 1.1, and

$$
r_{K, \sigma}(f) \leqq \int_{K}\left(\int_{X}|f| d v\right) d \sigma(v)=\int_{X}|f| d \mu .
$$

If $\mu \in M_{+}(X)$, then there exists a measurable function $\alpha: X \rightarrow[0,1]$ such that $\{\alpha \geqq \varepsilon\}$ is compact for all $\varepsilon>0$, and

$$
\int_{X} \frac{1}{\alpha} d \mu<\infty \text {. }
$$

Let $\psi(x):=\alpha(x) \delta_{x}$, where $\delta_{x}$ is the one point measure in $x$, then $\psi$ is a Borel map from $X$ into $M_{+}(X), \psi(X)$ is uniformly tight and $K:=\overline{\psi(X)}$ is therefore $w^{*}$-compact and uniformly tight. Let $d \lambda:=(1 / \alpha) d \mu$ and $\sigma:=\lambda \circ \psi^{-1}$, then $\sigma$ is a finite positive Radon measure on $M(X)$, and

$$
\begin{aligned}
q_{\mu}(f) & =\int_{X}|f| d \mu=\int_{X} \alpha(x)|f(x)| d \lambda(x) \\
& =\int_{X}\left|\int_{X} f(y) d(\psi(x))(y)\right| d \lambda(x)=\int_{K}\left|\int_{X} f d v\right| d\left(\lambda \circ \psi^{-1}\right)(v) \\
& =\int_{K}\left|\int f d v\right| d \sigma(v) .
\end{aligned}
$$

This shows that $\left\{q_{\mu}\right\}$ and $\left\{r_{K, \sigma}\right\}$ generate the same topology.

We shall need a fourth topology on $C(X)$. This is the socalled strict topology on $C(X)$, which we denote by $\beta$. The strict topology is generated by the seminorms

$$
p_{\alpha}(f):=\|\alpha f\|_{X}=\sup _{x \in X}|\alpha(x) f(x)|
$$


where $\alpha$ runs through all bounded measurable functions on $X$ which vanish at infinity, i.e. $\{|\alpha| \geqq \varepsilon\}$ is relatively compact for all $\varepsilon>0$. This topology was first introduced by C. R. Buck for locally compact spaces and later generalized by many authors to general completely regular Hausdorff spaces (see e.g. [4]).

From Theorem 2 in [4] we know that $w \cong \beta \cong m$, and from Corollary 1.2 it follows easily that $w \subseteq \tau \subseteq \beta$, therefore we have

$$
w \subseteq \tau \subseteq \beta \cong m
$$

and we shall leave to the reader to prove that $\tau \neq w$ and $\tau \neq \beta$ if $X$ is infinite.

From Theorem 3 in [6] one easily deduces the following form of Riesz' representation theorem:

Theorem 1.3. (D. Pollard and F. Topsøe [6]). Let $\lambda: C_{+}(X) \rightarrow[0, \infty[$ be additive and suppose that $\lambda$ satisfies

(1.3.1) $\forall \varepsilon>0 \exists \delta>0 \exists C$ compact $\leqq X$ such that $\lambda(f) \leqq \varepsilon$ whenever $0 \leqq f \leqq 1$ and $f \leqq \delta$ on $C$.

Then there exists a unique measure $\mu \in M_{+}(X)$ representing $\lambda$, that is,

$$
\lambda(f)=\int_{X} f d \mu, \quad \forall f \in C_{+}(X) .
$$

Remark. Note that (1.3.1) holds if there exists $\left\{f_{n}\right\} \cong C_{+}(X)$ with the following two properties:

$$
\left\{f_{n} \leqq 1\right\} \text { is compact for all } n \geqq 1,
$$

$$
\lim _{n \rightarrow \infty} \lambda\left(f_{n}\right)=0 \text {. }
$$

\section{Concentration of $L_{0}$ or $L_{X}$.}

Let $X$ be a completely regular Hausdorff space and $\varphi$ a completely monotone function on $C_{+}(X)$, with representing measure $\xi$. We shall give necessary and sufficient conditions for $\xi$ to be concentrated on $L_{0}$ or $L_{X}$. First we need a measurability lemma:

LEMMA 2.1. If $Y$ is a Borel subset of the Stone-Cech compactification $\beta X$, then $L_{Y}$ is a Borel subset of $L$. The subset $L_{0}$ is open in $L$.

Proof. From $L_{0}=\{\lambda \in L \mid \lambda(1)<\infty\}$ follows that $L_{0}$ is open. Identifying $L_{0}$ with $M_{+}(\beta X)$, see (1.7), we get

$$
L_{Y}=\left\{\tilde{\mu} \in M_{+}(\beta X) \mid \tilde{\mu}(\beta X \backslash Y)=0\right\}
$$


and from the fact that $\tilde{\mu} \mapsto \tilde{\mu}(\beta X \backslash Y)$ is Borel on $M_{+}(\beta X)$ by Proposition 1 in [5] we deduce that $L_{Y}$ is Borel in $L_{0}$, hence in $L$.

Theorem 2.2. Let $\varphi: C_{+}(X) \rightarrow \mathrm{R}$ be completely monotone with representing measure $\xi$ (see (1.8.)). Then the following 3 statements are equivalent:

$$
\begin{gathered}
\xi\left(L \backslash L_{0}\right)=0, \\
\lim _{t \rightarrow 0} \varphi(t f)=\varphi(0), \quad \forall f \in C_{+}(X), \\
\lim _{t \rightarrow 0} \varphi(t)=\varphi(0),
\end{gathered}
$$

where $t$ also denotes the constant function equal to $t$.

Proof. (2.2.1) $\Rightarrow(2.2 .2)$ : Let $f \in C_{+}(X)$ and define $F_{t}(\lambda):=e^{-t \lambda(f)}$. Then

$$
0 \leqq F_{t}(\lambda) \leqq 1 \text { and } \quad \lim _{t \rightarrow 0} F_{t}(\lambda)=1
$$

for all $\lambda \in L_{0}$. Hence the assumption implies that

$$
\varphi(t f)=\int_{L_{0}} F_{t}(\lambda) d \xi(\lambda) \rightarrow \xi\left(L_{0}\right)=\varphi(0),
$$

as $t$ tends to zero.

(2.2.2) $\Rightarrow(2.2 .3)$ : Obvious.

$(2.2 .3) \Rightarrow(2.2 .1)$ : Let $F_{t}(\lambda)$ be defined as above but with $f$ replaced by the constant 1. If $\lambda \in L \backslash L_{0}$ then $\lambda(1)=\infty$, therefore we get

$$
\lim _{t \rightarrow 0} F_{t}(\lambda)=1_{L_{0}}(\lambda) \quad \text { for all } \lambda \in L .
$$

Hence by assumption

$$
\xi(L)=\varphi(0)=\lim _{t \rightarrow 0} \int_{L} F_{t}(\lambda) d \xi(\lambda)=\xi\left(L_{0}\right)
$$

and so $\xi\left(L \backslash L_{0}\right)=0$.

TheOREM 2.3. Let $\varphi: C_{+}(X) \rightarrow R$ be a completely monotone function with representing measure $\xi$, and let $\varrho$ be a topology on $C(X)$ satisfying $\tau \cong \varrho \subseteq \beta$. Then the following 6 statements are equivalent:

(2.3.1) $\exists Y \sigma$-compact $\cong X$ such that $\dot{\xi}\left(L \backslash L_{Y}\right)=0$,

(2.3.2) $\xi(L)=\sup \left\{\xi(K) \mid K \cong M_{+}(X)\right.$ compact and uniformly tight $\}$,

(2.3.3) $\varphi$ is uniformly $\varrho$-continuous,

(2.3.4) $\varphi$ is $\varrho$-continuous at 0 , 
(2.3.5) $\varphi \mid B$ is $\varrho$-continuous at 0 , where $B=\{f \in C(X) \mid 0 \leqq f \leqq 1\}$,

(2.3.6) $\forall \varepsilon>0 \exists \delta>0 \exists C$ compact $\leqq X$ such that $\varphi(0)-\varphi(f) \leqq \varepsilon$ whenever $f \in B$ and $f \leqq \delta$ on $C$.

Note that we identify $M_{+}(X)$ with $L_{X}$ in (2.3.2) (see (1.6)).

Proof. (2.3.1) $\Rightarrow$ (2.3.2): First we note that $L_{Y} \cong L_{X} \cong L_{0}$ and therefore $\xi\left(L \backslash L_{0}\right)=0$. Let $\left\{C_{n}\right\}$ be compact sets in $X$ with $C_{1}=\varnothing$ and $C_{n} \uparrow Y$; then we may define

$$
F_{n}(v):=v\left(\beta X \backslash C_{n}\right) \quad \text { for } v \in L_{0}=M_{+}(\beta X) .
$$

Then $F_{n}: L_{0} \rightarrow\left[0, \infty\left[\right.\right.$ is Borel and $\lim _{n \rightarrow \infty} F_{n}(v)=0$ for all $v \in L_{Y}$. From Lemma 2.1 we know that $L_{Y} \in \mathscr{B}(L)$, and since $\xi\left(L \backslash L_{Y}\right)=0$ by assumption, we have that $F_{n} \rightarrow 0$ a.e. [ $\xi$ ]. Hence by Egoroff's theorem we can find for any given $\varepsilon>0$ a sequence $a_{1} \geqq a_{2} \geqq \ldots \geqq 0$ of positive numbers such that

$$
\begin{gathered}
\lim _{n \rightarrow \infty} a_{n}=0, \\
\xi\left(\left\{v \mid F_{n}(v) \leqq a_{n}, \forall n \geqq 1\right\}\right) \geqq \xi(L)-\varepsilon .
\end{gathered}
$$

Now since $F_{n}$ is lower semi-continuous on $L_{0}$ (see Proposition 1 in [5]) and $F_{1}(v)=v(\beta X)$, we have that

$$
K:=\left\{v \in L_{0} \mid F_{n}(v) \leqq a_{n}, \forall n \geqq 1\right\}
$$

is a compact uniformly tight subset of $M_{+}(X)$ with $\xi(K) \geqq \xi(L)-\varepsilon$. Hence (2.3.2) holds.

(2.3.2) $\Rightarrow$ (2.3.3): Given $\varepsilon>0$ choose $K \cong M_{+}(X)$ compact and uniformly tight so that $\xi(K) \geqq \xi(L)-\varepsilon / 4$. We claim that $|\varphi(f)-\varphi(g)|<\varepsilon$ whenever $r_{K, \xi}(|f-g|)<\varepsilon / 2$ (see (1.9) for the definition of $r_{K, \xi}$ ). In fact, if $r_{K, \xi}(|f-g|)<\varepsilon / 2$ for two functions $f, g \in C_{+}(X)$, then

$$
\begin{aligned}
|\varphi(f)-\varphi(g)| & \leqq \varphi(f)-\varphi(f \vee g)+\varphi(g)-\varphi(f \vee g) \\
& =\int_{L}\left(e^{-\lambda(f)}-e^{-\lambda(f \vee g)}\right) d \xi(\lambda)+\int_{L}\left(e^{-\lambda(g)}-e^{-\lambda(f \vee g)} d \xi(\lambda)\right. \\
& \leqq \int_{L}\left[1-e^{-\lambda(f \vee g-f)}\right] d \xi(\lambda)+\int_{L}\left[1-e^{-\lambda(f \vee g-g)}\right] d \xi(\lambda) \\
& \leqq \frac{\varepsilon}{2}+\int_{K} \lambda(f \vee g-f) d \xi(\lambda)+\int_{K} \lambda(f \vee g-g) d \xi(\lambda) \\
& =\frac{\varepsilon}{2}+\int_{K} \lambda(2(f \vee g)-f-g) d \xi(\lambda)
\end{aligned}
$$




$$
\begin{aligned}
& =\frac{\varepsilon}{2}+\int_{K} \lambda(|f-g|) d \xi(\lambda) \\
& =\frac{\varepsilon}{2}+r_{K, \xi}(|f-g|)<\varepsilon .
\end{aligned}
$$

This shows that $\varphi$ is uniformly $\tau$-continuous. (2.3.3) follows because $\tau$ is weaker than $\varrho$ by assumption.

(2.3.3) $\Rightarrow(2.3 .4) \Rightarrow(2.3 .5)$ : Obvious.

(2.3.5) $\Rightarrow(2.3 .6)$ : Since $\varrho$ is weaker than $\beta$, we have that $\varphi \mid B$ is $\beta$-continuous at 0 . Let $\chi$ be the topology on $C(X)$ of uniform convergence on compact subsets of $X$, then by Proposition 1 in [4], $\varkappa$ coincides with $\beta$ on $B$. Hence $\varphi \mid B$ is $x$-continuous at 0 and this evidently implies (2.3.6).

(2.3.6) $\Rightarrow(2.3 .1)$ : First we note that $(2.3 .6)$ implies that $\lim _{t \rightarrow 0} \varphi(t)=\varphi(0)$, therefore $\xi\left(L \backslash L_{0}\right)=0$ by Theorem 2.1. Now let

$$
M_{n}:=\left\{v \in L_{0} \mid v(1) \leqq n\right\} .
$$

Let $f \in C_{+}(X)$ and define

$$
F_{t}(\lambda):=\frac{1}{t}\left(1-e^{-t \lambda(f)}\right) \quad \text { for } t>0, \lambda \in L_{0} .
$$

Then we have

$$
\begin{gathered}
\lim _{t \rightarrow 0} F_{t}(\lambda)=\lambda(f) \quad \forall \lambda \in L_{0} \\
0 \leqq F_{t}(\lambda) \leqq \lambda(f) \leqq\|f\|_{X} \lambda(1)
\end{gathered}
$$

and this implies that

$$
\mu_{n}(f):=\int_{M_{n}} \lambda(f) d \xi(\lambda)=\lim _{t \rightarrow 0} \int_{M_{n}} F_{t}(\lambda) d \xi(\lambda)
$$

for all $f \in C_{+}(X)$. Let for $A \in \mathscr{B}(\beta X)$

$$
\tilde{\mu}_{n}(A):=\int_{M_{n}} \lambda(A) d \xi(\lambda)
$$

then by Lemma $1.1 \tilde{\mu}_{n}$ is a positive Radon measure on $\beta X$ with

$$
\mu_{n}(f)=\int_{\beta X} f d \tilde{\mu}_{n} \quad \forall f \in C(X) .
$$

Now we use the elementary inequality

$$
x \leqq(1+a)\left(1-e^{-x}\right) \quad \text { for } 0 \leqq x \leqq a
$$


to conclude that

$$
F_{t}(\lambda) \leqq \lambda(f) \leqq(n+1)\left(1-e^{-\lambda(f)}\right)
$$

for $f \in B$ and $\lambda \in M_{n}$. Hence we get

$$
\mu_{n}(f) \leqq(n+1) \int_{M_{n}}\left(1-e^{-\lambda(f)}\right) d \xi(\lambda) \leqq(n+1)(\varphi(0)-\varphi(f))
$$

for all $f \in B$. The assumption (2.3.6) now implies that $\mu_{n}$ satisfies (1.3.1) and by Theorem 1.3 we have, that $X$ is $\tilde{\mu}_{n}$-measurable and $\tilde{\mu}_{n}(\beta X \backslash X)=0$.

Hence we can find a $\sigma$-compact subset $Y \subseteq X$ such that $\tilde{\mu}_{n}(\beta X \backslash Y)=0$ for all $n \geqq 1$. But then we have

$$
\xi\left(\left\{\lambda \in M_{n} \mid \lambda(\beta X \backslash Y)>0\right\}\right)=0 \quad \forall n \geqq 1
$$

and since $M_{n} \uparrow L_{0}$ and $\xi\left(L \backslash L_{0}\right)=0$, we finally get

$$
\xi\left(L \backslash L_{Y}\right)=\xi\left(L_{0} \backslash L_{Y}\right)=\xi\left(\left\{\lambda \in L_{0} \mid \lambda(\beta X \backslash Y)>0\right\}\right)=0
$$

which proves our theorem.

\section{The Lévy continuity theorem on $M_{+}(X)$.}

Let $X$ be a completely regular Hausdorff space. Then $M_{+}\left(M_{+}(X)\right)$ denotes the set of positive finite Radon measures on $\left(M_{+}(X), w^{*}\right)$, and $M_{t}\left(M_{+}(X)\right)$ denotes the set of all $\sigma \in M_{+}\left(M_{+}(X)\right)$ satisfying

$$
\sigma\left(M_{+}(X)\right)=\sup \{\sigma(K) \mid K \text { compact and uniformly tight }\} .
$$

Note that $M_{t}\left(M_{+}(X)\right)=M_{+}\left(M_{+}(X)\right)$ if $X$ is semi-Radonian (see Theorem 10 in [5]).

If $\sigma \in M_{+}\left(M_{+}(X)\right)$, then we define its Laplace transform $\hat{\sigma}$ by

$$
\hat{\sigma}(f):=\int_{M_{+}(X)} \exp \left(-\int_{\mathrm{X}} f d v\right) d \sigma(v)
$$

for $f \in C_{+}(X)$. Note that $\hat{\sigma}$ is completely monotone on $C_{+}(X)$. If $X$ is $\sigma$ compact, then the set of all Laplace transforms of measures on $M_{+}(X)$ is characterised by those completely monotone functions $\varphi$ on $C_{+}(X)$ which satisfy one of the continuity properties (2.3.3)-(2.3.6) stated in Theorem 2.3.

We shall consider $M_{+}\left(M_{+}(X)\right)$ and $M_{t}\left(M_{+}(X)\right)$ equipped with their weak topologies, coming from the space $C\left(M_{+}(X), w^{*}\right)$. Let $\psi$ denote the map $M_{+}(X) \rightarrow L$ given by (1.6), and let

$$
\Psi(\sigma):=\sigma \circ \psi^{-1}
$$


be the corresponding map from $M_{+}\left(M_{+}(X)\right)$ to $M_{+}(L)$. It is easily checked that

(3.2) $\Psi$ is a homeomorphism of $M_{+}\left(M_{+}(X)\right)$ onto

$$
M_{X}(L):=\left\{\xi \in M_{+}(L) \mid \xi^{*}\left(L \backslash L_{X}\right)=0\right\}
$$

(see e.g. Corollary 9 in $[3$, p. 244]).

THEOREM 3.1. Let $\left\{\sigma_{\alpha}\right\}$ be a net in $M_{+}\left(M_{+}(X)\right)$ satisfying

(3.1.1) $\sup _{\alpha} \sigma_{\alpha}\left(M_{+}(X)\right)<\infty$,

(3.1.2) $\hat{\sigma}_{\alpha}(f) \rightarrow \varphi(f)$ for all $f \in C_{+}(X)$, where $\varphi \mid B$ is $\beta$-continuous at 0 , $B:=\{f \in C(X) \mid 0 \leqq f \leqq 1\}$.

Then there exists a measure $\sigma \in M_{t}\left(M_{+}(X)\right)$ whose Laplace transform is $\varphi$ and $\sigma_{\alpha} \rightarrow \sigma$ weakly.

Proof. Let $A:=\sup _{\alpha} \sigma_{\alpha}\left(M_{+}(X)\right)$ and let

$$
M_{A}:=\left\{\xi \in M_{+}(L) \mid \xi(L) \leqq A\right\} .
$$

Then $\xi_{\alpha}:=\Psi\left(\sigma_{\alpha}\right) \in M_{A}$ for all $\alpha$, and $M_{A}$ is a compact subset of $M_{+}(L)$. If $\xi$ is a limit point of $\left\{\xi_{\alpha}\right\}$, then

$$
\begin{aligned}
\hat{\xi}(f) & =\int_{L} e^{-\lambda(f)} d \xi(\lambda)=\lim _{\alpha} \int_{L} e^{-\lambda(f)} d \xi_{\alpha}(\lambda) \\
& =\lim _{\alpha} \hat{\sigma}_{\alpha}(f)=\varphi(f) .
\end{aligned}
$$

Hence $\hat{\xi}$ is a completely monotone function on $C_{+}(X)$, with representing measure $\xi$. Since a measure on $L$ is uniquely determined by its Laplace transform (see Corollary 2.5 of [1]), we find that $\left\{\xi_{\alpha}\right\}$ admits at most one limit point in $M_{+}(L)$. Hence $\xi=\lim _{\alpha} \xi_{\alpha}$ exists and $\hat{\xi}=\varphi$.

Then by (3.1.2) and Theorem 2.3 we conclude that $\xi=\Psi(\sigma)$ for some $\sigma \in M_{t}\left(M_{+}(X)\right)$, and $\hat{\sigma}=\varphi$. Therefore by (3.2) we find that $\sigma_{\alpha} \rightarrow \sigma$ weakly.

THeOREM 3.2. Let $\mathscr{K}$ be a subset of $M_{+}^{1}\left(M_{+}(X)\right)$, the probability Radon measures on $M_{+}(X)$. Let again $B:=\left\{f \in C_{+}(X) \mid 0 \leqq f \leqq 1\right\}$. Then we have

(i) If $\{\hat{\sigma}|B| \sigma \in \mathscr{K}\}$ is $\beta$-equicontinuous at 0 , then $\mathscr{K}$ is a relatively compact subset of $M_{t}\left(M_{+}(X)\right)$.

(ii) If $\mathscr{K}$ is uniformly tight and $X$ is a Prohorov space (see e.g. [5] for this notion), then $\{\hat{\sigma}|B| \sigma \in \mathscr{K}\}$ is $\beta$-equicontinuous at 0 .

Proof. (i). Follows immediately from Theorem 3.1. 
(ii). Let $\varepsilon>0$ be given. There exists by assumption a compact set $K \cong M_{+}(X)$ such that

$$
\sup \left\{\sigma\left(M_{+}(X) \backslash K\right) \mid \sigma \in \mathscr{K}\right\}<\frac{\varepsilon}{3} .
$$

$X$ being a Prohorov space we can find a compact subset $C$ of $X$ such that

$$
\sup \{v(X \backslash C) \mid v \in K\}<\frac{\varepsilon}{3} .
$$

From the compactness of $K$ we deduce that $A:=\sup \{v(X) \mid v \in K\}$ is finite. Now suppose that $f \in B, f \mid C<\varepsilon / 3 A$ and $\sigma \in \mathscr{K}$. Then w's get

$$
1-\hat{\sigma}(f)=\int_{M_{+}(X)}\left(1-e^{-\lambda(f)}\right) d \sigma(\lambda) \leqq \frac{\varepsilon}{3}+\int_{K}\left(1-e^{-\lambda(f)}\right) d \sigma(\lambda)
$$

and for $\lambda \in K$

$$
1-e^{-\lambda(f)} \leqq \lambda(f) \leqq \frac{\varepsilon}{3}+\int_{C} f d \lambda \leqq \frac{2 \varepsilon}{3}
$$

hence $1-\hat{\sigma}(f) \leqq \varepsilon$, showing the required $\beta$-equicontinuity at 0 .

The next theorem might be more useful for applications. Note that if $\sigma \in M_{+}\left(M_{+}(X)\right)$, then its Laplace transform is defined in a natural way on all non-negative Borel functions on $X$, in particular on Borel subsets of $X$.

THEOREM 3.3. Let $\mathscr{K} \cong M_{+}^{1}\left(M_{+}(X)\right)$ satisfy the following two conditions (3.3.1) $\lim _{A \rightarrow \infty} \sup _{\sigma \in \mathscr{X}} \sigma\left(\left\{v \in M_{+}(X) \mid v(C)>A\right\}\right)=0, \forall C \cong X$ compact, (3.3.2) $\lim \sup (1-\hat{\sigma}(X \backslash C))=0$, C $\sigma \in \mathscr{X}$

where the limit is taken along the net of compact subsets of $X$. Then $\mathscr{K}$ is a relatively compact subset of $M_{+}\left(M_{+}(X)\right)$.

Proof. Let $0<\varepsilon<1,0<\delta<1$; then $1-e^{-\delta} \geqq \frac{1}{2} \delta$ and

$$
\begin{aligned}
1-\hat{\sigma}(X \backslash C) & =\int_{M_{+}(X)}\left(1-e^{-\lambda(X \backslash C)}\right) d \sigma(\lambda) \\
& \geqq \int_{\left\{\lambda \mid \lambda(X \backslash C) \geqq \frac{1}{2} \delta\right\}}\left(1-e^{-\lambda(X \backslash C)}\right) d \sigma(\lambda) \geqq \frac{\delta}{4} \sigma\left(\left\{\lambda \mid \lambda(X \backslash C) \geqq \frac{\delta}{2}\right\}\right) .
\end{aligned}
$$


By (3.3.2) there exists a compact set $C \cong X$ such that

$$
\sup _{\sigma \in \mathscr{X}} \frac{\delta}{4} \sigma\left(\left\{\lambda \mid \lambda(X \backslash C) \geqq \frac{\delta}{2}\right\}\right) \leqq \frac{\varepsilon \delta}{24}
$$

hence

$$
\inf _{\sigma \in \mathscr{X}} \sigma\left(\left\{\lambda \mid \lambda(X \backslash C)<\frac{\delta}{2}\right\}\right) \geqq 1-\frac{\varepsilon}{6}
$$

and applying (3.3.1) we find $A \in \mathrm{R}$ such that

$$
\inf _{\sigma \in \mathscr{X}} \sigma(\{\lambda \mid \lambda(C) \leqq A\}) \geqq 1-\frac{\varepsilon}{6} .
$$

Putting

$$
L_{1}:=\left\{\lambda \in M_{+}(X) \mid \lambda(X \backslash C)<\frac{\delta}{2} \text { and } \lambda(C) \leqq A\right\}
$$

we have

$$
\inf _{\sigma \in \mathscr{X}} \sigma\left(L_{1}\right) \geqq 1-\frac{\varepsilon}{3}
$$

Now let $f \in C_{+}(X), 0 \leqq f \leqq 1$ and $\sup _{x \in C} f(x)<\varepsilon / 3 A$. Then for any $\sigma \in \mathscr{K}$ we get

$$
1-\hat{\sigma}(f)=\int_{M_{+}(X)}\left(1-e^{-\lambda(f)}\right) d \sigma(\lambda) \leqq \frac{\varepsilon}{3}+\int_{L_{1}} \lambda(f) d \sigma(\lambda)
$$

and for $\lambda \in L_{1}$

$$
\lambda(f)=\int_{X} f d \lambda \leqq \frac{\delta}{2}+\int_{C} f d \lambda \leqq \frac{\delta}{2}+\frac{\varepsilon}{3} .
$$

Hence, choosing $\delta=\frac{2}{3} \varepsilon, \quad 1-\hat{\sigma}(f) \leqq \varepsilon$, proving $\beta$-equicontinuity of $\{\hat{\sigma}|B| \sigma \in \mathscr{K}\}$ at 0 . From Theorem 3.2 we get the desired conclusion.

\section{Completely alternating functions on $C_{+}(X)$.}

A class of functions on $C_{+}(X)$, closely connected to completely monotone functions, is that of completely alternating (or alternating of infinite order) functions. A function $\psi: C_{+}(X) \rightarrow[0, \infty]$ is completely alternating if and only if 


$$
\sum_{i=1}^{n} \sum_{j=1}^{n} c_{i} c_{j} \psi\left(f_{i}+f_{j}\right) \leqq 0
$$

for all $n \in \mathrm{N}, f_{1}, \ldots, f_{n} \in C_{+}(X)$ and $c_{1}, \ldots, c_{n} \in \mathrm{R}$ such that $\sum_{i=1}^{n} c_{i}=0$, (see [1, Proposition 3.2 and Theorem 4.2]). One of the main results in [1] was the "Lévy-Khinchin"-representation for completely alternating functions (Theorem 3.7 in [1]). This uniquely determined representation has the form

$$
\psi(f)=c+h(f)+\int_{L \backslash\{0\}}\left(1-e^{-\lambda(f)}\right) d \xi_{0}(\lambda)
$$

where $c \in\left[0, \infty\left[, h: C_{+}(X) \rightarrow\left[0, \infty\left[\right.\right.\right.\right.$ is additive and $\xi_{0}$ is a non-negative Radon-measure on $L \backslash\{0\}$. Observing that

$$
L \backslash\{0\}=\{\lambda \in L \mid \lambda(1)>0\}
$$

we can write this representation in the folowing form

$$
\psi(f)=c+\int_{\beta X} \tilde{f} d x+\int_{L \backslash\{0\}} \frac{1-e^{-\lambda(f)}}{1-e^{-\lambda(1)}} d \xi(\lambda)
$$

where $x \in M_{+}(\beta X), \xi \in M_{+}(L)$ and

$$
\Delta_{1} \psi(f):=\psi(f+1)-\psi(f)=\int_{L} e^{-\lambda(f)} d \xi(\lambda),
$$

cf. the proof of Theorem 3.7 in [1].

Note that each completely alternating function $\psi$ on $C_{+}(X)$ satisfies the inequalities

$$
\begin{array}{cc}
\alpha \psi(f) \leqq \psi(\alpha f) & \forall f \in C_{+}(X), \forall \alpha \in[0,1] \\
\psi(\beta f) \leqq \beta \psi(f) & \forall f \in C_{+}(X), \forall \beta \in[1, \infty[.
\end{array}
$$

This follows from (4.1) and from the fact that

$$
\begin{array}{cc}
1-e^{-\alpha \lambda} \geqq \alpha\left(1-e^{-\lambda}\right) & \forall \lambda \geqq 0, \forall \alpha \in[0,1] \\
1-e^{-\beta \lambda} \leqq \beta\left(1-e^{-\lambda}\right) & \forall \lambda \geqq 0, \forall \beta \in[1, \infty[
\end{array}
$$

which is easily established using Cauchy's mean value theorem. Another important property is subadditivity

$$
\psi(f+g) \leqq \psi(f)+\psi(g) \quad \forall f, g \in C_{+}(X),
$$

cf. Proposition 3.5 in [1].

THEOREM 4.1. Let the completely alternating function $\psi: C_{+}(X) \rightarrow[0, \infty[$ have the representation (4.1). Then $\xi\left(L \backslash L_{0}\right)=0$ if and only if $\lim _{t \rightarrow 0} \psi(t)=\psi(0)$. 
Proof. We may and do assume $\psi(0)=c=0$. Suppose that $\lim _{t \rightarrow 0} \psi(t)=0$. By (4.4)

hence

$$
0 \leqq \psi(t+1)-\psi(1) \leqq \psi(t) \rightarrow 0 \quad \text { as } t \rightarrow 0,
$$

$$
\Delta_{1} \psi(t)=\psi(t+1)-\psi(t) \rightarrow \psi(1)=\Delta_{1} \psi(0)
$$

and we get $\xi\left(L \backslash L_{0}\right)=0$ from Theorem 2.2. The other direction follows immediately from (4.1).

TheOREM 4.2. Let $\psi: C_{+}(X) \rightarrow[0, \infty[$ be a completely alternating function with the representation (4.1); let $\varrho$ be any topology on $C(X)$ satisfying $\tau \cong \varrho \subseteq \beta$ and put

$$
B:=\{f \in C(X) \mid 0 \leqq f \leqq 1\} .
$$

Then the following 5 statements are equivalent:

(4.2.1) $\exists Y \sigma$-compact $\cong X$ such that $\chi(X \backslash Y)=0$ and $\xi\left(L \backslash L_{Y}\right)=0$

(4.2.2) $\quad \psi \mid B$ is $\tau$-continuous at 0

(4.2.3) $\psi \mid B$ is uniformly $\varrho$-continuous

(4.2.4) $\quad \psi \mid B$ is $\beta$-continuous at 0

(4.2.5) $\forall \varepsilon>0 \exists \delta>0 \exists C$ compact $\leqq X$ such that $\psi(f)-\psi(0) \leqq \varepsilon$ whenever $f \in B$ and $f \leqq \delta$ on $C$.

Proof. Again we assume $\psi(0)=c=0$.

(4.2.1) $\Rightarrow$ (4.2.2): The function $f \mapsto \int_{\beta X} \tilde{f} d x$ is $\tau$-continuous because $x \in M_{+}(X)$, cf. Corollary 1.2. By Theorem 2.2 there exist compact uniformly tight subsets $K_{n} \subseteq L_{Y} \backslash\{0\}$ such that $\xi\left(L \backslash K_{n}\right) \leqq 1 / n$. We define

$$
\psi_{n}(f):=\int_{K_{n}} \frac{1-e^{-\lambda(f)}}{1-e^{-\lambda(1)}} d \xi(\lambda), \quad f \in C_{+}(X), n \in \mathrm{N} .
$$

By Theorem $2.3\left\{\psi_{n}\right\}$ is a sequence of $\tau$-continuous completely alternating functions. Now

$$
\sup _{f \in B} \frac{1-e^{-\lambda(f)}}{1-e^{-\lambda(1)}} \leqq 1 \quad \text { for all } \lambda \in L
$$

implying that $\psi_{n}$ converges uniformly to $\psi(f)-\int f d x$ on $B$. Hence $\psi \mid B$ is $\tau$ continuous.

(4.2.2) $\Rightarrow(4.2 .3)$ : If $f, g$ belong to $C_{+}(X)$ then applying the subadditivity (4.4) we have

$$
\begin{aligned}
& |\psi(f)-\psi(g)| \leqq \psi(f \vee g)-\psi(f)+\psi(f \vee g)-\psi(g) \\
& \leqq \psi\left((f-g)^{+}\right)+\psi\left((g-f)^{+}\right) .
\end{aligned}
$$


If now $\psi \mid B$ is $\tau$-continuous at 0 , then $\psi \mid B$ is uniformly $\tau$-continuous, as one can see immediately from the definition of the $\tau$-topology.

(4.2.3) $\Rightarrow$ (4.2.4): Obvious.

(4.2.4) $\Rightarrow(4.2 .5)$ : This can be seen as in Theorem 2.3.

(4.2.5) $\Rightarrow(4.2 .1)$ : Let $\varepsilon>0$ and choose $\delta>0, C \cong X$ compact such that $\psi(f)$ $<\varepsilon$ for all $f \in B, f \leqq \delta$ on $C$. For those $f$ we get

$$
\Delta_{1} \psi(0)-\Delta_{1} \psi(f)=\psi(1)-\psi(f+1)+\psi(f) \leqq \psi(f)<\varepsilon
$$

hence there exists by Theorem 2.3 a $\sigma$-compact subset $Y_{1} \leqq X$ such that $\xi\left(L \backslash L_{Y_{1}}\right)=0$. The function $f \mapsto \int_{\beta X} \tilde{f} d x$ has of course also the continuity property of (4.2.5), hence by Theorem 1.3., $\varkappa$ belongs to $M_{+}(X)$ and therefore is concentrated on a $\sigma$-compact subset $Y_{2} \cong X$. The union $Y:=Y_{1} \cup Y_{2}$ fulfills condition (4.2.1).

\title{
REFERENCES
}

1. C. Berg, J. P. R. Christensen and P. Ressel, Positive definite functions on abelian semigroups, Math. Ann. (to appear).

2. G. Choquet, Theory of capacities, Ann. Inst. Fourier (Grenoble), 5 (1954), 131-295.

3. J. Hoffmann-Jørgensen, The theory of analytic spaces, Århus University, Various Publications Series No. 10 (1970).

4. J. Hoffmann-Jørgensen, A generalization of the strict topology, Math. Scand. 30 (1972), 313-323.

5. J. Hoffmann-Jørgensen, Weak compactness and tightness of subsets of $M(X)$, Math. Scand. 31 (1972), 127-150.

6. D. Pollard and F. Topsøe, A unified approach to Riesz type representation theorems, Studia Math. 54 (1975), 173-190.

7. F. Topsøe, Topology and measure, Lecture Notes in Mathematics 133, Springer-Verlag, Berlin $\cdot$ Heidelberg $\cdot$ New York, 1970.

\author{
UNIVERSITY OF ARHUS \\ DENMARK \\ AND \\ UNIVERSITY OF FREIBURG/BR. \\ WEST-GERMANY
}

